\title{
Successful treatment of Chlamydophila pneumoniae acute respiratory distress syndrome with extracorporeal membrane oxygenator: a case report and diagnostic review
}

David De Bels ${ }^{1 *}$, Philippe Gottignies ${ }^{1}$, Marijke Reynders ${ }^{2}$, Sébastien Roques ${ }^{1}$, Stephan Wilmin ${ }^{1}$, Véronique-Yvette Miendje Deyi ${ }^{3}$, Sophie Jamart ${ }^{1}$ and Jacques Devriendt ${ }^{1}$

\begin{abstract}
Introduction: Chlamydophila pneumoniae is a respiratory pathogen known to infect the upper and lower respiratory tracts. Infection severity can range from sub-clinical pulmonary infection to acute respiratory distress syndrome.

Case presentation: A previously healthy 62-year-old Caucasian man was admitted to our hospital for acute respiratory failure. Serum samples obtained every week starting from the day of admission showed clear-cut seroconversion for $\mathrm{C}$. pneumoniae antibodies. All other cultures obtained during the first days of hospitalization were negative. Despite maximal ventilatory support (high positive end expiratory pressure, fraction of inspired oxygen of 1.0, nitric oxide inhalation, neuromuscular blocking agents and prone positioning), our patient remained severely hypoxemic, which led us to initiate an extracorporeal membrane oxygenation treatment. Extracorporeal membrane oxygenation and hemodiafiltration were withdrawn on day 12. Our patient was extubated on day 18 and discharged from our Intensive Care Unit on day 20. He went home a month later.

Conclusion: We describe the first published case of acute respiratory distress syndrome due to C. pneumoniae infection successfully treated by extracorporeal membrane oxygenation, a very useful tool in this syndrome. A quick and specific method for the definite diagnosis of Chlamydophila infection should be developed.
\end{abstract}

\section{Introduction}

Chlamydophila pneumoniae is an obligate intracellular Gram-negative bacterium. The spectrum of disease, in addition to pneumonia and influenza-like illness, includes pharyngitis, sinusitis, bronchitis, exacerbation of chronic obstructive pulmonary diseases and reactive arthritis [1-5]. C. pneumoniae accounts for $6 \%$ to $20 \%$ of cases of community-acquired pneumonia (CAP) [1,2]. Many of these cases have few symptoms and don't require hospitalization ('walking pneumonia'). However, more severe cases may occur, with up to $18 \%$ requiring hospitalization [6] and even mechanical ventilation, especially in elderly, immunocompromised hosts and

\footnotetext{
* Correspondence: david.debels@chu-brugmann.be

${ }^{1}$ Intensive Care Department, Brugmann University Hospital, 4 Place Van

Gehuchten, 1020, Brussels, Belgium

Full list of author information is available at the end of the article
}

patients with coexisting cardiopulmonary disease [7], but also, rarely, in previously healthy adults [8].

Old and new macrolides are effective against C. pneumoniae and have been recommended as first-line treatment. New fluoroquinolones are also effective in vitro against C. pneumoniae and can be used. Studies have shown that $35 \%$ to $47 \%$ of C. pneumoniae pneumonia is mixed with other pathogens, the most common being Streptococcus pneumoniae $[9,10]$.

We describe the case of severe CAP due to C. pneumoniae infection in a previously healthy adult patient, with acute respiratory distress syndrome (ARDS) necessitating extracorporeal membrane oxygenation (ECMO).

\section{Case presentation}

A previously healthy 62-year-old Caucasian man was admitted to our hospital for acute respiratory failure.

\section{C) Biomed Central}


Our patient developed a fever of up to $40^{\circ} \mathrm{C}$ seven days earlier and a non-productive cough three days later. $\mathrm{He}$ had not received any antimicrobial treatment prior to his hospitalization, the diagnosis of his primary care physician being influenza $(\mathrm{A} / \mathrm{H} 1 \mathrm{~N} 1 \mathrm{v})$, given the ongoing outbreak.

His medical history was remarkable for possible viral pericarditis without any consequence in 2007 and a gastric ulcer 30 years earlier. He had no drinking habits. He did not smoke cigarettes. He had not travelled abroad recently. He did not have any bird or pet.

On hospital admission, our patient was in acute respiratory distress. His respiratory rate was 40 breaths/ minute, his temperature $38.3^{\circ} \mathrm{C}$, his pulse 98 beats/minute and his blood pressure 114/60 mmHg. Auscultation revealed crackles over his whole left lung and over his right lower lung field. A computed tomography scan showed diffuse alveolar type infiltrates in his two lung fields with air bronchograms (Figure 1).

Arterial blood gas analysis (under 100\% oxygen through a non-rebreathing mask) showed $\mathrm{pH} 7.54$, a partial pressure of carbon dioxide $\left(\mathrm{PaCO}_{2}\right) 44 \mathrm{mmHg}$, partial pressure of oxygen $\left(\mathrm{PaO}_{2}\right) 38 \mathrm{mmHg}$ and an arterial blood oxygen saturation of $84 \%$. His white blood cell count was 5780 cells/ $\mu \mathrm{L}$ (86\% neutrophils) and the erythrocyte sedimentation rate was $92 \mathrm{~mm} / \mathrm{h}$.
Laboratory values showed serum creatinine at $1.7 \mathrm{mg} /$ $\mathrm{dL}$, potassium at $2.8 \mathrm{mEq} / \mathrm{L}$, creatine phosphokinase at 644 IU/L, liver test alterations (alanine transaminase at $87 \mathrm{IU} / \mathrm{L})$, lactate dehydrogenase elevation (1708 IU/L) and D-Dimers at $7420 \mathrm{ng} / \mathrm{mL}$, activated partial thromboplastin time of 72 seconds, normal international normalized ratio and blood platelets at $166.000 / \mu \mathrm{L}$. His urine output was $0.4 \mathrm{~mL} / \mathrm{kg} / \mathrm{h}$ over six hours. An electrocardiogram showed a sinus tachycardia with a complete right bundle branch block.

Serum samples obtained every week as from the day of admission showed a clear-cut seroconversion for $C$. pneumoniae antibodies (the course of the antibody titers shown in Table 1).

Paired serum samples and antigens against the most common microorganisms, including atypical bacteria and common viruses such as $\mathrm{A} / \mathrm{H} 1 \mathrm{~N} 1 \mathrm{v}$, were negative. Blood, sputum and urine tests for bacterial cultures obtained during the first day of hospitalization were negative.

Our patient was treated by amoxycillin-clavulanic acid, moxifloxacin and oseltamivir. His respiratory status necessitated endotracheal intubation and mechanical ventilation. Severe arterial hypotension prompted norepinephrine infusion and the insertion of a pulmonary artery catheter. The initial hemodynamic pattern was

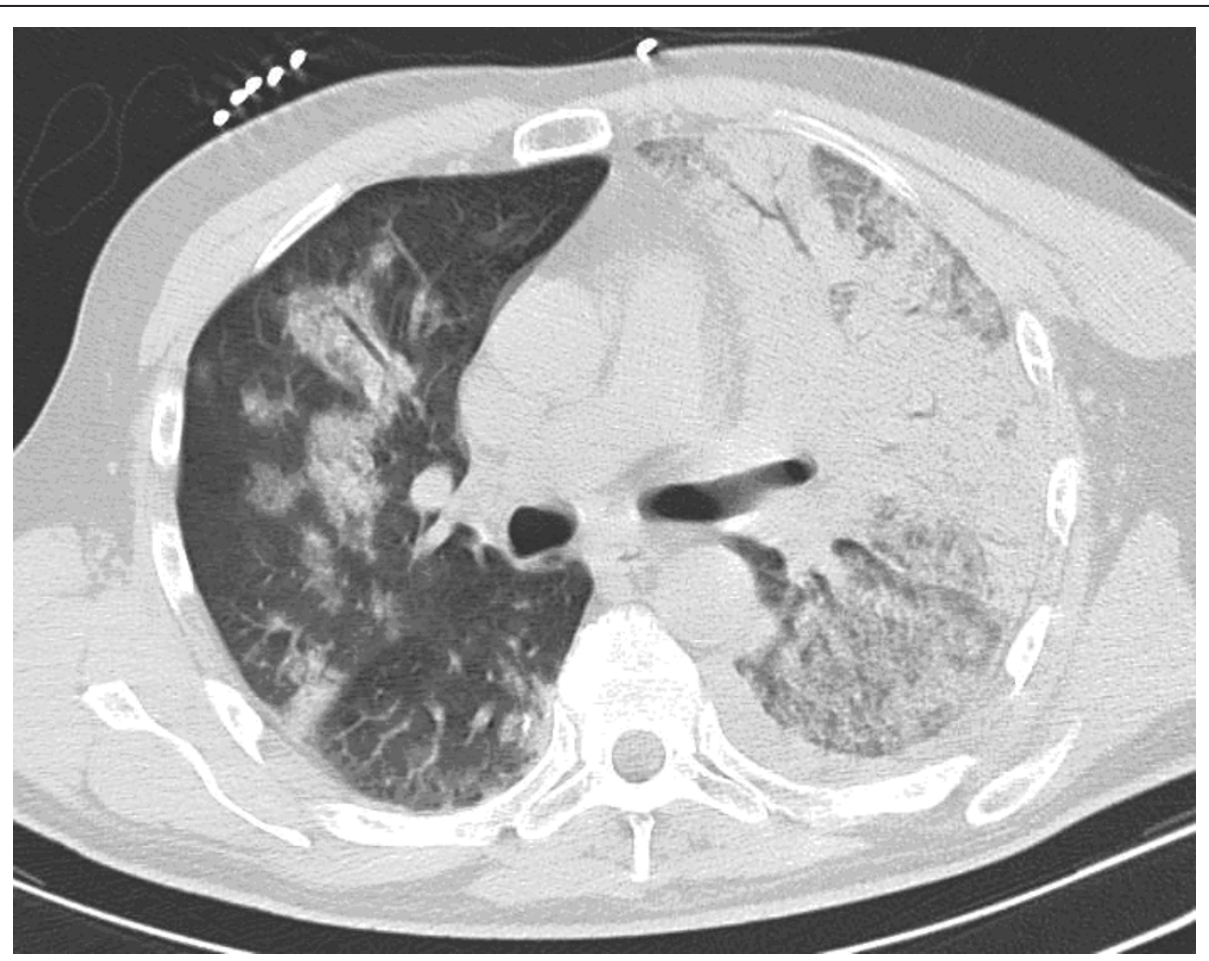

Figure 1 Unenhanced computed tomography scan through the thorax showing alveolar type infiltrates of the two lung field with air bronchograms. 
Table 1 Comparison of antibody kinetics using different techniques

\begin{tabular}{lrrr}
\hline Test (units) & Day one & Day 13 & Day 23 \\
\hline MIF-lgG $^{\text {a }}$ (titer) & $1 / 512$ & $1 / 2048$ & $1 / 4096$ \\
MIF-lgM $^{\text {b }}$ (titer) & $<1 / 10$ & $<1 / 10$ & $<1 / 10$ \\
MOMP-IgG $^{c}(\mathrm{AU} / \mathrm{mL})$ & 102 & 478 & $>500$ \\
LPS-lgG $^{\text {d }}$ (index) & 1.5 & 6.8 & 9.4 \\
LPS-IgA $^{\text {e }}$ (index) & $<0.9$ & 2.8 & 1.3 \\
\hline
\end{tabular}

${ }^{a}$ MIF-IgG (Focus Diagnostics): + when $>1 / 512$ or a four-fold rise in titer in consecutive sera; ${ }^{\mathrm{b}} \mathrm{MIF-IgM}$ (Focus Diagnostics): $<1 / 16=$ negative; ${ }^{\mathrm{c}} \mathrm{MOMP}$-lgG (C. pneumoniae IgG-ELISA plus medac): + when $\geq 28 \mathrm{AU} / \mathrm{mL} ;{ }^{\mathrm{d}} \mathrm{LPS}-\lg \mathrm{G}(\mathrm{C}$. pneumoniae IgG- rELISA medac): + when index $\geq 1,1$; ${ }^{\mathrm{L}} \mathrm{LPS}-\mathrm{IgA}$ (C. pneumoniae IgA- rELISA medac): + when index $\geq 1,5$; doubtful: index 0.9-1.4.

typical for sepsis (hemodynamic values are shown in Table 2). Metabolic data showed a mixed venous saturation of $56 \%$.

Despite maximal ventilatory support (high positive end expiratory pressure, an inspired oxygen fraction $\left(\mathrm{FiO}_{2}\right)$ of 1.0, nitric oxide inhalation of $20 \mathrm{ppm}$, neuromuscular blocking agents and prone positioning), our patient remained severely hypoxemic $\left(\mathrm{PaO}_{2} / \mathrm{FiO}_{2}=38\right)$ which led us to initiate ECMO treatment.

Venovenous ECMO (a Sorin Revolution centrifugal pump, a Sorin ECCO oxygenator and a Sorin Satcrit console from Sorin Group, Milano, Italy) was put in place on the fifth day of hospitalization, with a left femoral 22-Fr drainage cannula and a right femoral 23Fr return cannula, inducing a drastic improvement of our patient's oxygenation parameters $\left(\mathrm{PaO}_{2}=120\right.$ $\mathrm{mmHg})$. The mixed venous oxygen saturation $\left(\mathrm{SVO}_{2}\right)$ increased from $56 \%$ to $86 \%$. Continuous veno-venous hemodiafiltration (CVVHDF) renal replacement therapy was also initiated on day three because of acute renal failure.

There were no severe complications of the ECMO treatment except for hemorrhagic suffusion of the two femoral catheter insertion points, requiring a blood transfusion.

ECMO and CVVHDF were withdrawn on day 12. Our patient was extubated on day 18 and discharged from

Table 2 Hemodynamic values throughout Intensive Care Unit stay till ECMO removal.

\begin{tabular}{lrrrr}
\hline Parameters & Day one & Day five & Day nine & Day 12 \\
\hline HR & 97 & 88 & 92 & 104 \\
MAP & 60 & 67 & 69 & 91 \\
PCWP & 18 & 12 & 7 & 6 \\
CI (3.0-5.0) & 3.9 & 4.2 & 2.7 & 4.6 \\
ISVR (1200-2000) & 861 & 1416 & 2414 & 1512 \\
Noradrenaline $(\mu \mathrm{g} / \mathrm{kg} / \mathrm{min})$ & 1.1 & 0.6 & 0.2 & 0 \\
Dobutamine $(\mu \mathrm{g} / \mathrm{kg} / \mathrm{min})$ & 15 & 20 & 5 & 0 \\
\hline
\end{tabular}

HR: heart rate (beats per min); MAP: mean arterial pressure $(\mathrm{mmHg})$; PCWP: pulmonary capillary wedge pressure $\left(\mathrm{cmH}_{2} \mathrm{O}\right) ; \mathrm{Cl}$ : cardiac index $\left(\mathrm{L} / \mathrm{min} / \mathrm{m}^{2}\right)$; indexed systemic vascular resistance (Dyne.sec. $\mathrm{m}^{2} / \mathrm{cm}^{5}$ ). our Intensive Care Unit on day 20. He went home a month later. He is now in good physical condition and has returned to work and to a normal social life.

\section{Discussion}

This case illustrates the polymorphism in the presentation of C. pneumoniae infection, which can cause severe CAP complicated by ARDS, even in immunocompetent patients. Pneumonia and bronchitis are the most common clinical infections associated with C. pneumoniae. The classical pulmonary presentation is a single subsegmental infiltrate, even though lobar consolidation or bilateral infiltrates can also be seen.

Two major aspects are discussed below: the rationale for the use of ECMO in ARDS patients as well as its different techniques, and the difficulties of diagnosing $C$. pneumoniae pneumonia.

\section{ECMO and $\mathrm{CO}_{2}$ removal}

There are two main ECMO techniques according to the type of vascular access that is used: venovenous and venoarterial. Each one has a specific indication. Venoarterial ECMO gives cardiac and respiratory support. Indications for venoarterial ECMO include postcardiac surgery (heart surgery or heart transplantation), cardiogenic shock due to acute myocardial infarction or acute myocarditis and intoxication. For cardiac support, the goal is to optimize organ perfusion (by obtaining a $\mathrm{SVO}_{2}$ greater than $70 \%$, which usually needs an output index of about $3 \mathrm{~L} /$ $\mathrm{min} / \mathrm{m}^{2}$ ). This is achieved by choosing the appropriate sizes of cannula according to the patient's body surface area.

In isolated respiratory failure, a venovenous access is preferred. The objective is $\mathrm{CO}_{2}$ removal at least equal to the patient's metabolism (roughly $3 \mathrm{~cm}^{3} / \mathrm{kg} / \mathrm{min}$ in adults). The purpose of venovenous ECMO use in ARDS patients is lung protection (reducing ventilatorinduced lung injury) through a decrease in alveolar distention permitted by the reduction in ventilator conditions.

Even though a lot of progress has been done in technical issues, the risk-benefit ratio must be taken into account when ECMO is proposed to a patient with severe respiratory failure. As in all extracorporeal devices, anticoagulation is mandatory. Many patients will experience bleeding, which can be very severe. Contraindication for anticoagulation remains the most important limitation of this technique. Previous severe disability or poor prognosis due to underlying disease constitutes the other main contraindication for ECMO initiation. The main complications are bleeding, thromboembolism, cannula-related complications, pulmonary embolism or infarction, aortic thrombosis and coronary 
or cerebral hypoxemia; the latter three being more frequent in a venoarterial montage type.

Discussion still stands in the literature as to whether mechanical ventilation of more than seven days is or is not a relative contraindication for ECMO because of ventilation-induced lung injury [11].

The first randomized clinical trials failed to demonstrate beneficial effect of ECMO for severe respiratory failure in the 1970s and 1980s. Since then, technical improvements for ECMO on one hand, and better treatment of the ARDS (protective ventilation and others) on the other hand, have renewed interest in ECMO [12]. The recent CESAR trial (Conventional Ventilation or ECMO for Severer Adult Respiratory Failure [13]) demonstrated a possible beneficial effect of ECMO. Of those patients referred for ECMO, there was 63\% survival rate at six months without disability, compared to $47 \%$ in those who were assigned to conventional management. This translates to one extra survivor without disability for every six patients treated.

A recent Italian experience, in patients with ARDS due to the influenza A/H1N1 virus, based on pre-emptive patient centralization showed a $77 \%$ survival rate if ECMO was started within seven days of initiation of mechanical ventilation [11]. Indication for ECMO in this study was refractory hypoxia or an oxygenation index below 30 despite a $\mathrm{PaO}_{2} / \mathrm{FiO}_{2}$ ratio greater than $100 \mathrm{mmHg}$.

Treatment of critically ill patients affected by the 2009 Influenza A (H1N1v) outbreak in Australia and New Zealand [14] included ECMO, with a 71\% survival rate at Intensive Care Unit discharge, an excellent result. These three studies emphasize the renewed place of ECMO in the treatment of severe ARDS with very good survival rates, considering the severity of the initial insult.

The limitation of plateau airway pressures and the low tidal volume used in ARDS patients have been at the cost of an increased $\mathrm{PaCO}_{2}$. There has therefore been an increase in interest for extracorporeal $\mathrm{CO}_{2}$ removal techniques. Different techniques have been developed but most of them have not gone to relevant, sufficiently powered clinical trials due to technical problems or insufficient $\mathrm{CO}_{2}$ removal or oxygenation [15]. The pumpless extracorporeal lung assist (PECLA), or Novalung ${ }^{\mathbb{B}}$ [Novalung $\mathrm{GmbH}$, Heilbronn, Germany], is a compact pumpless device driven by the pressure gradient between arterial and venous blood. A femorofemoral setting is most commonly used. The main advantage of a pumpless device is a reduction in mechanical blood trauma, bleeding and hemolysis. The Novalung ${ }^{\circledR}$ has been widely studied in ARDS. Three studies have demonstrated the clinical efficacy of this PECLA device [16-18]. $\mathrm{CO}_{2}$ removal is efficient but oxygenation may be insufficient in these critically ill patients.

\section{C. pneumoniae diagnosis}

Diagnosis of C. pneumoniae pneumonia is still debated in the current literature. According to Grayston et al. and Saikku et al. [19,20], who first described this Chlamydia species, almost everybody is infected and reinfected with C. pneumoniae throughout his or her life. Unspecific symptoms of C. pneumoniae infections make the diagnosis even more difficult, with a possible underestimation of its frequency. Undetected infections may lead to chronic disease with serious consequences, such as atherosclerotic cardiovascular disease [21] or asthma. Its incidence in CAP ranges from $3 \%$ to $22 \%$, varying according to the diagnostic method used [22]. The most frequent routinely used diagnostic techniques are serological, including complement fixation test, immunofluorescence assay, microimmunofluorescence (MIF) and genus- and species-specific enzyme-linked immunosorbent assay (ELISA) systems. No currently available serologic tests of a single serum specimen will provide conclusive evidence of a current infection with $C$. pneumoniae.

Since the publication of the Centre for Disease Control (CDC) 2001 recommendations for Nucleic Acid Amplification Tests (NAATs) [23], a multitude of inhouse polymerase chain reaction (PCR) tests have been described, though very few of them have been validated by the CDC. Results of NAATs may be unreliable because of cross-contamination, inappropriate treatment of the clinical samples (leading to the loss of the target nucleic acid) or the presence of inhibiting substances [24]. Additionally, validation of these tests is primarily analytical and not against clinically obtained specimens.

Bacterial culture has traditionally been considered the 'gold standard' for diagnosis; however, its sensitivity, even in excellent laboratories, seldom exceeds $90 \%$ and is typically between $75 \%$ and $85 \%$. The culture is technically difficult to implement and is only available in a few laboratories worldwide [25].

Even though MIF is still actually considered as the 'serological gold standard' technique for the detection of species-specific antibodies, there is a large discrepancy between MIF testing for C. pneumonia and detection of these organisms by culture or PCR. The MIF test is reliable for detection of a prior exposure to Chlamydiae by the presence of immunoglobulin G (IgG) antibodies and is relatively sensitive for the detection of IgM. IgM is an unreliable marker of acute infection in adolescents and adults since it is often not present, presumably because of previous infection by a chlamydial species [24]. Additionally, MIF is quite a laborious and subjective 
technique that requires much experience, is not standardized and has significant laboratory-to-laboratory variations.

In our institution, in order to get better sensitivity (for example, after reinfections), better reproducibility, greater objectivity and less cross-reactivity in the serology tests for Chlamydiaceae, two-level serological testing has been implemented for C. pneumoniae. The first level test is a search by ELISA-technique for anti-major outer membrane protein (MOMP) IgG and IgA antibodies (C. pneumoniae-IgG \& IgA-ELISA plus, Medac, Hamburg, Germany) which are species-specific. This permits us to screen patients. The disadvantage of antiMOMP antibodies is their late appearance in acute primary infection (three weeks for IgA, six to eight weeks for IgG) and their considerable persistence in human serum afterwards, independent of the bacterial eradication. In general, the prevalence of C. pneumoniae IgG antibody reaches $50 \%$ in adults above 20 years of age, and $80 \%$ in the elderly population (> 70 years old). The second level test is another ELISA assay, rELISA (Medac), for the detection of genus-specific anti-lipopolysaccharide(LPS) antibodies. Chlamydiae contain, as a common immunodominant antigen, the LPS to which the first immune reaction is directed. These anti-LPSs have the main advantage of rising quickly during acute infection (five to ten days for IgA), allowing early diagnosis, and returning to normal a few weeks after the infection and are thus associated to the eradication of the bacterium [26]. The use of paired sera enables the discrimination of current infections, reinfections and reactivations (defined by titer increase) from chronic persistent ones (constant LPS, IgG and IgA antibody titers, present in $5 \%$ to $10 \%$ of the adult population) for which no antibacterial treatment is needed. The latter is due to the chronic presence of bacteria in the organism in a latent phase (monocytes). Criteria for acute infections are a four-fold increase in IgA or IgG or a doubling of IgA and IgG at 10 to 15 days.

Moreover, the described consecutive sera of our patient underwent an additional retrospective examination by a MIF assay (Chlamydia MIF IgG \& Chlamydia MIF IgM, Focus Diagnostics, Cypress, CA, USA). A straightforward IgG seroconversion could also be observed (Table 1). Thanks to the detection of IgA and of IgG antibodies to C. pneumoniae in various combinations, serology allows a classification of the state of infection. In our patient, reinfection was diagnosed because MIF were positive on admission; there was a four-fold increase in anti-MOMP IgG and, finally, an important rise and a rapid decline in anti-LPS IgA.

\section{Conclusion}

C. pneumoniae can induce very severe ARDS. We describe the first published case of ARDS due to $C$. pneumoniae infection successfully treated by ECMO. Definite Chlamydophila diagnosis remains a challenge but should be sought for in severe ARDS patients without evidence of other infectious cause. A quick and specific method for the definite diagnosis of Chlamydophila infections should be developed.

\section{Consent}

Written informed consent was obtained from the patient for publication of this case report and any accompanying images. A copy of the written consent is available for review by the Editor-in-Chief of this journal.

Funding

No funding was granted for this study.

\section{Author details}

${ }^{1}$ Intensive Care Department, Brugmann University Hospital, 4 Place Van Gehuchten, 1020, Brussels, Belgium. ${ }^{2}$ Microbiology Laboratory, AZ Sint Jan Ziekenhuis, 10 Ruddershove, 8000 Brugge, Belgium. ${ }^{3}$ Microbiology Laboratory, Brugmann University Hospital, 4 Place Van Gehuchten, 1020, Brussels, Belgium.

\section{Authors' contributions}

DDB, JD and PG have taken part in the conception, the design and the writing of the article. JD and SJ have acquired the data. SW has analyzed and interpreted the data. VYMD and MR have done all the microbiological analyses. JD, MR and SR have critically revisited it for important intellectual content. All authors read and approved the final manuscript.

\section{Competing interests}

The authors declare that they have no competing interests.

Received: 14 June 2011 Accepted: 17 January 2012

Published: 17 January 2012

\section{References}

1. Balin BJ, Gerard HC, Arking EJ, Appelt DM, Branigan PJ, Abrams JT, Whittum-Hudson JA, Hudson AP: Identification and localization of Chlamydia pneumoniae in the Alzheimer's brain. Med Microbiol Immunol 1998, 187:23-42.

2. Elkind MS, Lin IF, Grayston JT, Sacco RL: Chlamydia pneumoniae and the risk of first ischemic stroke: The Northern Manhattan Stroke Study. Stroke 2000, 31:1521-1525.

3. Hahn DL, Peeling RW: Airflow limitation, asthma, and Chlamydia pneumoniae-specific heat shock protein 60. Ann Allergy Asthma Immunol 2008, 101:614-618

4. Hoymans VY, Bosmans JM, leven MM, Vrints CJ: Chlamydia pneumoniaebased atherosclerosis: a smoking gun. Acta Cardiol 2007, 62:565-571.

5. Hunter SF, Hafler DA: Ubiquitous pathogens: links between infection and autoimmunity in MS? Neurology 2000, 55:164-165.

6. Lieberman D, Ben-Yaakov M, Lazarovich Z, Porath A, Schlaeffer F, Leinonen M, Saikku P, Horovitz O, Boldur I: Chlamydia pneumoniae community-acquired pneumonia: a review of 62 hospitalized adult patients. Infection 1996, 24:109-114.

7. Liu KT, Yang KY, Lee YC, Perng RP: Risk factor analysis of acute respiratory distress syndrome among hospitalized patients with Chlamydophila pneumoniae pneumonia. J Chin Med Assoc 2007, 70:318-323.

8. Gnarpe J, Gnarpe H, Nissen K, Haldar K, Naas J: Chlamydia pneumoniae infection associated with multi-organ failure and fatal outcome in a previously healthy patient. Scand J Infect Dis 1998, 30:523-524. 
9. File TM Jr, Plouffe JF Jr, Breiman RF, Skelton SK: Clinical characteristics of Chlamydia pneumoniae infection as the sole cause of communityacquired pneumonia. Clin Infect Dis 1999, 29:246-248.

10. Miyashita N, Fukano H, Okimoto N, Hara H, Yoshida K, Niki Y, Matsushima T: Clinical presentation of community-acquired Chlamydia pneumoniae pneumonia in adults. Chest 2002, 121:1776-1781.

11. Patroniti N, Zangrillo A, Pappalardo F, Peris A, Cianchi G, Braschi A, lotti GA, Arcadipane A, Panarello G, Ranieri VM, Terragni P, Antonelli M, Gattinoni L, Oleari F, Pesenti A: The Italian ECMO network experience during the 2009 influenza $A(\mathrm{H} 1 \mathrm{~N} 1)$ pandemic: preparation for severe respiratory emergency outbreaks. Intensive Care Med 2011, 37:1447-1457.

12. Patroniti N, Bellani G, Pesenti A: Nonconventional support of respiration. Curr Opin Crit Care 2011, 17:527-532.

13. Peek GJ, Mugford M, Tiruvoipati R, Wilson A, Allen E, Thalanany MM, Hibbert CL, Truesdale A, Clemens F, Cooper N, Firmin RK, Elbourne D, CESAR trial collaboration: Efficacy and economic assessment of conventional ventilatory support versus extracorporeal membrane oxygenation for severe adult respiratory failure (CESAR): a multicentre randomised controlled trial. Lancet 2009, 374:1351-1363.

14. Australia and New Zealand Extracorporeal Membrane Oxygenation (ANZ ECMO) Influenza Investigators, Davies A, Jones D, Bailey M, Beca J, Bellomo R, Blackwell N, Forrest P, Gattas D, Granger E, Herkes R, Jackson A, McGuinness S, Nair P, Pellegrino V, Pettilä V, Plunkett B, Pye R, Torzillo P, Webb S, Wilson M, Ziegenfuss M: Extracorporeal membrane oxygenation for 2009 influenza $\mathrm{A}(\mathrm{H} 1 \mathrm{~N} 1)$ acute respiratory distress syndrome. JAMA 2009, 302:1888-1895.

15. Lynch JE, Hayes D Jr, Zwischenberger JB: Extracorporeal CO(2) removal in ARDS. Crit Care Clin 2011, 27:609-625.

16. Florchinger B, Philipp A, Klose A, Hilker M, Kobuch R, Rupprecht L, Keyser A, Puhler T, Hirt S, Wiebe K, Müller T, Langgartner J, Lehle K, Schmid C: Pumpless extracorporeal lung assist: a 10-year institutional experience. Ann Thorac Surg 2008, 86:410-417, discussion 417.

17. Liebold A, Philipp A, Kaiser M, Merk J, Schmid FX, Birnbaum DE: Pumpless extracorporeal lung assist using an arterio-venous shunt. Applications and limitations. Minerva Anestesiol 2002, 68:387-391.

18. Walles T: Clinical experience with the iLA Membrane Ventilator pumpless extracorporeal lung-assist device. Expert Rev Med Devices 2007, 4:297-305.

19. Grayston JT, Diwan VK, Cooney M, Wang SP: Community- and hospitalacquired pneumonia associated with Chlamydia TWAR infection demonstrated serologically. Arch Intern Med 1989, 149:169-173.

20. Saikku P, Leinonen M, Mattila K, Ekman MR, Nieminen MS, Makela PH, Huttunen JK, Valtonen V: Serological evidence of an association of a novel Chlamydia, TWAR, with chronic coronary heart disease and acute myocardial infarction. Lancet 1988, 2:983-986.

21. Kalayoglu MV, Libby P, Byrne Gl: Chlamydia pneumoniae as an emerging risk factor in cardiovascular disease. JAMA 2002, 288:2724-2731.

22. Hammerschlag MR: Chlamydia pneumoniae and the lung. Eur Respir J 2000, 16:1001-1007.

23. Dowell SF, Peeling RW, Boman J, Carlone GM, Fields BS, Guarner J, Hammerschlag MR, Jackson LA, Kuo CC, Maass M, Messmer TO, Talkington DF, Tondella ML, Zaki SR, C. pneumoniae Workshop Participants: Standardizing Chlamydia pneumoniae assays: recommendations from the Centers for Disease Control and Prevention (USA) and the Laboratory Centre for Disease Control (Canada). Clin Infect Dis 2001, 33:492-503.

24. Loens $\mathrm{K}$, Goossens $\mathrm{H}$, leven $\mathrm{M}$ : Acute respiratory infection due to Mycoplasma pneumoniae: current status of diagnostic methods. Eur J Clin Microbiol Infect Dis 2010, 29:1055-1069.

25. Kumar $\mathrm{S}$, Hammerschlag MR: Acute respiratory infection due to Chlamydia pneumoniae: current status of diagnostic methods. Clin Infect Dis 2007, 44:568-576

26. Persson K, Haidl S: Evaluation of a commercial test for antibodies to the chlamydial lipopolysaccharide (Medac) for serodiagnosis of acute infections by Chlamydia pneumoniae (TWAR) and Chlamydia psittaci. APMIS 2000, 108:131-138.

doi:10.1186/1752-1947-6-20

Cite this article as: De Bels et al: Successful treatment of Chlamydophila pneumoniae acute respiratory distress syndrome with extracorporeal membrane oxygenator: a case report and diagnostic review. Journal of Medical Case Reports 2012 6:20.

\section{Submit your next manuscript to BioMed Central and take full advantage of:}

- Convenient online submission

- Thorough peer review

- No space constraints or color figure charges

- Immediate publication on acceptance

- Inclusion in PubMed, CAS, Scopus and Google Scholar

- Research which is freely available for redistribution 